\title{
G

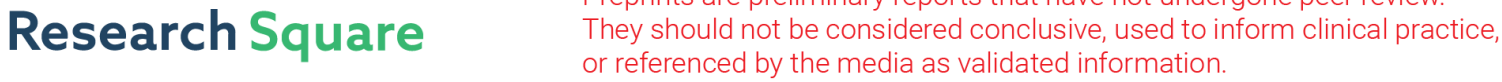 \\ Expression and prognostic roles PRDXs gene family in hepatocellular carcinoma
}

\section{Mingxing $X u$}

Third Affiliated Hospital of Sun Yat-Sen University

\section{Jianliang $\mathbf{X u}$}

Third Affiliated Hospital of Sun Yat-Sen University

\section{Dun Zhu}

Chaya People's Hospital

\section{Rishun Su}

Third Affiliated Hospital of Sun Yat-Sen University

\section{Baoding Zhuang}

Third Affiliated Hospital of Sun Yat-Sen University

Ruiyun Xu ( $\nabla$ sysuxuruiyun@163.com )

Third Affiliated Hospital of Sun Yat-Sen University

Lingli Li (D69064959@qq.com )

Banan District People's Hospital of Chongqing

Shuxian Chen ( $\square$ chenshx5@mail.sysu.edu.cn )

Third Affiliated Hospital of Sun Yat-Sen University

Yunbiao Ling ( $\square$ simdoctor@163.com )

the Third Affiliated Hoapital of Sun Yat-sen University https://orcid.org/0000-0002-5580-4159

\section{Research article}

Keywords: PRDX family, HCC, prognosis, bioinformatics analysis

Posted Date: March 26th, 2020

DOI: https://doi.org/10.21203/rs.3.rs-18264/v1

License: (c) (i) This work is licensed under a Creative Commons Attribution 4.0 International License.

Read Full License

Version of Record: A version of this preprint was published at Journal of Translational Medicine on March 26th, 2021. See the published version at https://doi.org/10.1186/s12967-021-02792-8. 


\section{Abstract}

Background: As the second leading cause of cancer-related death in the world, the therapeutic effect and 5-year overall survival of hepatocellular carcinoma (HCC) are not optimistic. Previous researches indicated that the disorder of PRDXs was related to the occurrence and development of cancer.

Methods: In this study, analyzing through CCLE, UALCAN, HCCDB and Human Protein Atlas database databases, PRDXs not only existed in various tumor cell lines, but also had maladjusted expression on mRNA and protein levels in HCC tissues. Besides, the correlations between mRNA as well as methylation levels of PRDXs and clinical characterization were evaluated using UALCAN.

Results: Kaplan-Meier Plotter demonstrated that high expression of PRDX1 or low expression of PRDX2/3 suggested poor prognosis for HCC patients. The mutation frequency, type and biological interaction network of PRDXs were obtained from c-Bioportal. By LinkedOmics, PRDXs related differential expressions genes were obtained, which were involved in several canonical pathways and certain amino acid metabolism, as well as miRNAs targeting PRDXs, some of which may effect on the progression of HCC.

Conclusions: In conclusion, the disordered expression of PRDXs was correlated with prognosis of HCC patients analyzing by online tools, suggesting that PRDXs could be new molecular targets for HCC treatment and prognosis prediction.

\section{Background}

Hepatocellular carcinoma (HCC) and intrahepatic cholangiocarcinoma (ICC) are two main types of primary hepatocellular carcinoma (PHC), of which HCC accounts for about $80 \%^{[1]}$. HCC can be caused by chronic infection of hepatitis B virus (HBV) or hepatitis C virus (HCV), alcoholism, metabolic syndrome associated with diabetes or obesity, and non-alcoholic fatty liver disease (NAFLD) ${ }^{[2]}$. In recent years, the incidence and mortality of HCC have been increasing in North America and several European regions, while in traditional high-risk areas, including Japan and parts of China, the incidence and mortality of HCC have been declining ${ }^{[3]}$. With approximately 800,000 deaths each year, HCC remains the second leading cause of cancer-related deaths worldwide ${ }^{[4]}$. The survival of HCC patients depends on a variety of factors, including Eastern Cooperative Oncology Group performance status, the presence of other complications, liver dysfunction, tumor burden, macrovascular invasion and metastasis ${ }^{[5]}$.

In developed countries, $40-50 \%$ of patients have been diagnosed with early HCC due to surveillance programs, and are in a stage of potentially curative treatment ${ }^{[2]}$. However, the possibility of metastasis is high even after potential radical treatment ${ }^{[6]}$, and the overall five-year survival rate after developing $\mathrm{HCC}$ is only $50 \%-70 \%[7]$. In addition, patients with advanced $\mathrm{HCC}$ at the time of diagnosis cannot undergo surgical resection, or thotopic liver transplantation, or local percutaneous tumor ablation ${ }^{[4]}$, and are not sensitive to chemotherapy or radiotherapy ${ }^{[8]}$. Therefore, patients with advanced HCC have a lower overall 
survival rate. Although the treatment of $\mathrm{HCC}$ has improved over the past decade, $\mathrm{HCC}$, as an aggressive malignant tumor, is still highly resistant and difficult to treat. Therefore, it is necessary to find novel molecular targets that can improve the treatment of HCC or predict the prognosis of HCC patients.

Peroxiredoxins (PRDXs) are now considered to be a major superfamily of peroxidases conserved during the evolution of bacteria, archaea and eukaryotes ${ }^{[9]}$. Six mammalian isoforms of PRDXs have been identified, all of which are 22-27 kDa small proteins ${ }^{[10]}$. PRDXs have constitutive expression in almost all tissues and cell types, although they differ in expression levels, and also exist in the extracellular environments, ${ }^{[9]}$. PRDXs can reduce peroxide through catalyzing the oxidation of cysteine to sulfonic acid $^{[11]}$. According to the position of cysteine residues in the catalytic reactions, they can be divided into three subgroups: typical 2-Cys: PRDX1-4; atypical 2-Cys: PRDX5 and 1-Cys: PRDX6 ${ }^{[10]}$. Among them, PRDX1, expressed in the cytoplasm of cells, is identified as the stress-induced macrophage protein produced by mouse peritoneal macrophages exposed to oxidative stress ${ }^{[12]}$. Extracellular PRDX1 plays a critical role in inflammatory regulation. It can be used as a molecular chaperone to regulate the actions of multitudinous molecules, or act as a regulator of transcription ${ }^{[13]}$. PRDX2, the peroxidase activity of which is activated by CDK2, inhibits the differentiation of acute myeloid leukemia (AML) cells ${ }^{[14]}$. PRDX4 controls neurogenesis by coupling the GDE2 surface expression in response to the redox environment in the endoplasmic reticulum ${ }^{[15]}$. Some research have shown that although PRDXs do not suppress cell proliferation, the loss of $\operatorname{Prdx} 1$ in mice results in tumorigenesis ${ }^{[10]}$.

Currently, there are few studies on the role of PRDXs in HCC and the prognosis of patients with HCC. In this study, we analyzed data from public databases via various online analysis tools to explore the expression and influence of PRDXs in HCC, especially its predictive role in the prognosis of HCC patients.

\section{Materials And Methods}

\section{CCLE analysis}

Cancer Cell Line Encyclopedia (CCLE) (https://portals.broadinstitute.org/ccle) provides public access to genomic data, analysis and visualization for 1457 cell lines. Each gene has multiple datasets and data identifiers. The five major dataset types are Copy Number, mRNA expression (Affy), RPPA, RRBS, and mRNA (RNAseq). In this study, the mRNA expression (RNAseq) data of PRDX family in a series of cancer cell lines were used.

\section{UALCAN analysis}

UALCAN (http://ualcan.path.uab.edu/analysis.html) is a comprehensive, friendly and interactive web resource to provide easy access to data from the Cancer Genome Atlas (TCGA) project. TCGA contains clinical data of 31 cancer types. The website can not only query and analyze the relative expression of genes between cancer samples and normal samples, but also can be based on the relative expression of individual cancer stage, tumor grade or other clinical pathological characteristics in different tumor 
subgroups. In this study, HCC samples from the TCGA were used to analyze the expression of PRDXs in $\mathrm{HCC}$ and normal tissues. The correlations between the expression of PRDXs and clinical pathological characteristics, including age, cancer stage, gender, and tumor grade were also accessed. Additionally, the correlations between the methylation levels of PRDXs and clinical pathological characteristics such as sample types, cancer stage and tumor grade were also evaluated.

\section{HCCDB analysis}

$\mathrm{HCCDB}$ is a web-based database, aiming at providing a one-stop resource for gene expression atlas in HCC. Fifteen public dataset sets of HCC gene expression were archived in the HCCDB database (http://lifeome.net/database/hccdb/home.html), including 3917 samples. The database can be used for the identification of consistently differentially expressed genes in $\mathrm{HCC}$, i.e. the function $t$-test in $\mathrm{R}$ is employed to detect whether there is significant difference in gene expression between tumor samples and adjacent samples in each dataset. Genes detected in at least 8 datasets and significantly differentially expressed in at least half of the datasets containing these genes are identified as uniformly differentially expressed. HCCDB database was used to confirm whether PRDXs were significantly differentially expressed in HCC.

\section{Human Protein Atlas analysis}

Human Protein Atlas (http://www.proteinatlas.org/) is a public database that allows free access to explore human proteome. It includes but not limited to Tissue Atlas, Cell Atlas and Pathology Atlas. Tissue Atlas consists of deep sequencing of RNA (RNAseq) from 37 major different normal tissue types and immunohistochemistry on tissue microarrays containing by 44 normal human tissue types. Cell Atlas provides a high-resolution insight into the spatial distribution of intracellular proteins, including mRNA expression profiles of 64 different human-derived cell lines, and details the subcellular distribution patterns of proteins in these cells. Pathology Atlas contains data on the expression of 17 major human cancer types from nearly 8,000 patients. In addition, the corresponding proteins are analyzed by immunohistochemistry (IHC) to form a total of more than 5 million IHC cancer tissue images. In this study, only the immunohistochemical staining results of PRDXs in normal and pathological HCC samples were utilized.

\section{Kaplan-Meier Plotter analysis}

The Kaplan-Meier plotter (http://kmplot.com/analysis/) is capable to assess the effect of 54,000 genes on survival in 21 cancer types. Gene expression data, relapse free and overall survival information are downloaded from GEO, EGA and TCGA. According to various quantile expressions of the proposed biomarkers, the patient samples are divided into two groups. Kaplan-Meier survival plot is used to compare the two groups. The hazard ratio of $95 \%$ confidence intervals and logrank $P$ value are calculated. In this study, the correlation between the mRNA levels of PRDX family members and overall survival of 364 patients with HCC was studied by Kaplan-Meier plotter. The hazard ratio (HR) and logrank $P$ were presented in the results. $P<0.05$ means statistical significance. 


\section{c-BioPortal analysis}

The cBio Cancer Genomics Portal (https://www.cbioportal.org/) is a comprehensive open resource, which can be used for interactive exploration of multiple cancer genomics datasets. Currently, there are 260 cancer studies and stores DNA copy number data (hypothesis and discrete values of each gene, such as "deep deletion" or "amplification" and $\log _{2}$ level), expression data of mRNA and miRNA, non-synonymous mutations, protein and phosphoprotein level (RPPA) data, DNA methylation data and some clinical data, etc. We analyzed genetic alterations of PRDXs in 372 HCC samples from TCGA. The search parameters included mutation, CNVs and mRNA expression. The tab OncoPrint shows an overview of genetic changes for each sample in PRDXs. The tab Network visualizes the biological interaction network of PRDXs in the public access database, and carries out color coding and screening options according to the frequency of genomic alterations of each gene.

\section{LinkedOmics analysis}

LinkedOmics (http://www.linkedomics.org/login.php) is a publicly available portal that includes multiomics data from 32 TCGA cancer types and mass spectrometry-based proteomics data generated by the Clinical Proteomics Tumor Analysis Consortium (CPTAC) for TCGA breast, colorectal and ovarian tumors. The web application has 3 analytical modules: LinkFinder, LinkInterpreter and LinkCompare. LinkFinder module was used to investigate differentially expressed genes related to PRDXs in TCGA LIHC (HCC) cohort $(n=371)$. Pearson Correlation test was performed for statistical analysis, and the results were presented in volcano plot and heat map. The data in LinkFinder results were signed and ranked. Then differentially expressed genes related to PRDXs were analyzed and enriched based on Kyoto Encyclopedia of Genes and Genomes (KEGG), Gene Ontology and other functional classifications by using GSEA of LinkInterpreter module. The rank criterion was false discovery rate (FDR) $<0.05$, and 500 simulations were carried out.

\section{Results}

\section{The expression of PRDXs in hepatocellular carcinoma in different databases}

The mRNA levels of PRDXs in various common cancer cell lines were obtained from CCLE database. It was found that the mRNA levels of PRDXs were maintained at 6-9, which was relatively higher compared with other cancer cell lines (Figure 1 and Supplementary figure 1-2). Furthermore, the expression levels of PRDXs in HCC tissues were accessed by UALCAN database and compared with that in normal liver tissues (Figure 2). PRDX1 (Figure 2A), PRDX2 (Figure 2B), PRDX5 (Figure 2E) and PRDX6 (Figure 2F) were significantly upregulated in liver cancer while PRDX4 (Figure 2D) was downregulated in HCC. Besides, it was observed that PRDX3 exhibited no differential expression between HCC tissues and normal liver tissues (Figure 2C).

What's more, the mRNA expression of PRDXs in HCC, adjacent normal tissue, cirrhotic and healthy samples was obtained from HCCDB database. PRDX1 was verified to be upregulated in HCC tissues 
compared with para-carcinoma tissues in all HCC datasets but HCCDB11 (Figure 3A), and PRDX5 was also upregulated in HCC tissues in all datasets except for HCCDB11 and HCCDB16 (Figure 3B). PRDX2 was attested to have higher expression levels in HCC tissues in HCCDB7 and HCCDB18 datasets

(Supplementary figure 3A). Moreover, the downregulation of both PRDX3 and PRDX4 in HCC tissues was illustrated by HCCDB1, HCCDB3, HCCDB4, HCCDB13, HCCDB15 and HCCDB16 dataset. Besides, PRDX3 was also downregulated in HCCDB6 (Supplementary figure 3B), and PRDX4 was downregulated in HCCDB12 (Supplementary figure 4A). Similarly, results of HCCDB1, HCCDB4, HCCDB6, HCCDB15 and HCCDB17 datasets indicated the downregulated expression levels of PRDX6 in HCC, and data of HCCDB7 dataset exhibited conversed results (Supplementary figure 4B).

The protein levels of PRDXs gene family in hepatocellular carcinoma and the correlation between PRDXs expression and clinical characteristics

The protein expression of PRDXs was collected from the immumohistochemical staining results of Human Protein Atlas database. It was obvious that the protein levels of PRDX1 (Figure 4A), PRDX2

(Figure 4B) and PRDX5 (Figure 4E) were significantly upregulated in HCC tissues, while that of PRDX3 (Figure 4C) and PRDX4 (Figure 4D) was downregulated. Moreover, there was no differential expression of PRDX6 between HCC tissues and normal liver tissues (Figure 4F). The correlation analysis between expression of PRDXs and age, cancer stage, gender, and tumor grade was further performed based on the data of TCGA database by UALCAN. Except for the upregulation of PRDXs in HCC patients compared with normal people, the significant association between expression of some members of PRDX gene family and age (Supplementary table 1), cancer stage (Supplementary table 2), gender (Supplementary table 3) and tumor grade (Supplementary table 4) can be observed. In addition, correlation analysis between the methylation levels of PRDXs in HCC and cancer stage as well as tumor grade was also carried out. The results showed that the methylation levels of PRDX1 and PRDX3 in HCC patients were obviously higher than that in normal people, whereas that of PRDX2/4/5 were lower (Supplementary table 5). Also, several significant correlation between methylation levels of PRDXs and cancer stage (Supplementary table 6) or tumor grade (Supplementary table 7) can be observed.

\section{mRNA expression of PRDXs and its association with the overall survival (OS) of patients with HCC}

According to the analysis results of Kaplan-Meier Plotter database, overexpression of PRDX1 was associated with a poor prognosis for patients with $\mathrm{HCC}(\mathrm{HR}=1.63)$ (Figure $5 \mathrm{~A})$. However, the lowly expressed of PRDX2 (Figure 5B) and PRDX3 (Figure 5C) led to the poor prognosis value of patients with HCC. The expression of other PRDX family members (PRDX4/5/6) was not significantly correlated with the survival of HCC patients (Figure 5D-F).

\section{The genomic alterations of PRDXs in HCC}

The frequency and types of PRDXs alterations in HCC based on sequencing data from patients with HCC in the TCGA database were monitored by cBioPortal (Figure 6A). The results revealed that PRDX1 and PRDX4 were both altered in 26 of 372 (7\%) patients with HCC. Besides, both of PRDX2 and PRDX5 were 
altered in 33 of $372(9 \%)$ patients with HCC. PRDX3 was altered in 40 of $372(11 \%)$ HCC patients, and $18 \%$ (63/372) of HCC patients exhibited PRDX6 alteration. Moreover, the specific types and frequency of PRDXs alterations in HCC showed in Table 1. These alterations were mRNA upregulation in 24 cases (6.4\%) of PRDX1, 30 cases (8.1\%) of PRDX2, 19 cases (5\%) of PRDX3, 31 cases (8.3\%) of PRDX5, and 33 cases (8.9\%) of PRDX6. Furthermore, the tab Network in cBioPortal was used to reflect interactions between PRDXs and the neighborhood genes in HCC (Figure 6B). The neighbor genes of PRDXs with the most frequent alterations were MYC (18.6\%), MAPK1 (13.1\%) and PBK (11.4\%) (Table 1).

\section{KEGG pathway analyses of co-expression genes correlated with PRDXs in HCC}

The $372 \mathrm{HCC}$ patients in the TCGA were used to analyze mRNA sequencing data by the LinkFinder module of LinkedOmics. As shown by the volcano plot in Figure 7A, 11082 genes showed negative correlation with PRDX1 and a total of 8840 genes were positive correlation with PRDX1. The top 50 of positive correlated significant genes presented in Figure 7B by heat map, whereas the top 50 of negative correlated significant genes showed in Figure $7 \mathrm{C}$ by heat map. There was a strong negative correlation between the expression of PHF2 and PRDX1 (Pearson correlation $=0.62, P=2.547 \mathrm{e}-40$ ), whereas PSMB2 was strongly positively correlated with PRDX1 (Pearson correlation $=0.72, P=1.961 \mathrm{e}-59$ ). In addition, the specific analysis results of genes that negatively or positively correlated with PRDX family members were shown in panels A-C of Supplementary figure 5-9. Combining all of the above results, it indicated that PRDXs had extensive influence on the transcriptome. Furthermore, the significant KEGG pathway analysis of genes differentially expression in correlation with PRDX1 by gene set enrichment analysis (GSEA) revealed enrichment in Metabolic pathways, TGF-beta signaling pathway, and so on (Figure 7D). It suggested that the enriched pathways of genes differentially expression in correlation with PRDX3, PRDX4 and PRDX5 were also involved in Metabolic pathways (panels D of Supplementary figure 6-8). To further investigate the miRNA targets of PRDX1 in HCC, the Linklnterpreter of LinkedOmics were utilized, and the results revealed that the network was associated with (GTCTTCC) MIR-7, (CTTTGTA) MIR-524, (TTGCACT) MIR-130A, MIR-301, MIR-130B (Figure 7E). Furthermore, miRNA targets networks of the other members of PRDX family were also analyzed and showed in panels E of Supplementary figure 5-9.

\section{Discussion}

Hepatocellular carcinoma (HCC) is a relatively common cancer with increasing morbidity and mortality. About half of the new cases and deaths of HCC occurring annually in China ${ }^{[16]}$. Despite various treatments, the prognosis of HCC patients is poor, with a 5-year overall survival rate of less than $50 \%$. However, through surgical excision or transplantation, the survival rate of HCC patients in early stage can reach $70 \%{ }^{[17]}$. The only chance for long-term survival is early detection of tumors. Unfortunately, most HCC patients are diagnosed as advanced and ineligible for treatment ${ }^{[18]}$.

It has been reported that the disorder of PRDX family was related to the occurrence and development of cancer ${ }^{[19]}$. To explore the predictive role of PRDXs in the prognosis of patients with HCC, the data from public databases were analyzed and evaluated in this study by various online tools. Firstly, according to 
the data from CCLE database, PRDXs were expressed in multiple cancer cell lines, and the levels of PRDXs were mainly between 6 and 9 in HCC cell lines. Knoops B et al. also found that PRDXs were expressed in almost all tissues and cell lines ${ }^{[9]}$. In addition, results of UALCAN database indicated that the mRNA expression of PRDX1/2, 5/6 in HCC was significantly up-regulated, while the mRNA expression of PRDX4 was down-regulated. Similar results were obtained from different HCC datasets of HCCDB database. What's more, immumohistochemical staining results in Human Protein Atlas database showed that PRDX1/2/5 were highly expressed in HCC tissues, while the expression of PRDX3/4 was low. This was similar to the results of Aguilar-Melero P et al. research, that is, PRDX1 was highly expressed in various tumors including $\mathrm{HCC}^{[20]}$. Silei Zhou et al. demonstrated that PRDX2 was often down-regulated in HCC tissues, and played a tumorigenic role ${ }^{[21]}$. Xiao Xu et al. found in clinical samples that the expression level of PRDX6 in HCC tissues was lower than that in matched para-carcinoma tissues ${ }^{[22]}$.

Kaplan-Meier survival analysis showed that high expression of PRDX1 and low expression of PRDX2/3 predicted poor prognosis of patients with HCC. There was no significant correlation between the expression of other PRDXs in HCC and prognosis. Previous studies have shown that PRDX1 overexpression was associated with poor clinical prognosis of $\mathrm{HCC}^{[20]}$, and Fang $\mathrm{Y}$ et al. also attested that decreased PRDX1 expression in tumor tissues was an independent risk factor for overall survival and disease free survival in patients after surgery ${ }^{[23]}$. The down-regulation of PRDX3 and PRDX6 expression was correlated with poor prognosis of HCC patients ${ }^{[22,24]}$. Although the relationship between PRDX2 and prognosis of HCC patients has not been reported, the low expression of PRDX2 was confirmed to be associated with liver metastasis and poor overall survival in colorectal cancer ${ }^{[25]}$. However, studies have shown that high expression of PRDX4 was associated with good tumor characteristics and prognosis of HCC patients ${ }^{[26]}$, which was inconsistent with our findings and needed to be validated in the experiments.

Furthermore, the types and frequency of PRDXs in HCC were determined based on sequencing data from HCC patients in the cBioPortal database. The maximum mutations of frequency was PRDX6 (18\%), and the minimum change of frequency was PRDX1 and PRDX4 (both 7\%). The tab network in cBioPortal was used to show the adjacent genes of PRDXs, frequency of which were changed, with MYC (18.6\%), MAPK1 (13.1\%) and PBK (11.4\%) having the most frequent changes in frequency. MYC is the main regulator of cell metabolism and proliferation, high constitutive expression of which drives many types of tumors, and often correlated with cancer invasion and poor prognosis ${ }^{[27]}$. Mitogen-activated protein kinase 1 (MAPK1), belonging to the MAP kinase family, is served as a binding site for many biochemical signals and involved in various cellular processes, such as cell differentiation, proliferation, transcriptional development and regulation ${ }^{[28]}$. PBK is a serine/threonine kinase belonging to the mitogen-activated protein kinase (MAPKK) family. PBK has a high trans-activity, plays a crucial role in various cancers, and also promotes migration and invasion of lung cancer cell ${ }^{[29]}$. In epithelial ovarian cancer, high expression levels of PBK are significantly associated with poor progression-free survival and overall survival in early stage patients ${ }^{[30]}$. 
LinkedOmics was employed to analyze the sequencing results of 372 patients with HCC in TCGA database. We found that the gene enrichment pathways associated with PRDXs mostly involved Metabolic pathway, as well as some classical pathways, such as NF-kappa B signaling pathway, TGFbeta signaling pathway and cell cycle. Study from Mingjun Zheng et al. revealed that PRDX1 knockdown significantly down-regulated the $\beta$-catenin level associated with WNT pathway, suggesting that PRDX1 knockdown inhibited the occurrence of EMT ${ }^{[31]}$. Rong Wang et al. suggested that PRDX2 served as a promoter of cancer stem cells properties in colon cancer via Hedgehog signaling pathway ${ }^{[2]}$. These results indicated that PRDXs had a extensive influence on the transcriptome and affected the progression of HCC through various downstream pathways. What's more, previous researches have manifested that PRDX2 acts as a target of miR-200b-3p, promoting proliferation, invasion and EMT of colorectal cancer

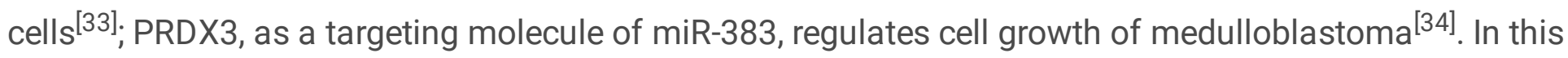
study, LinkInterpreter module of LinkedOmics was utilized to predict the interaction between PRDXs and miRNAs to preliminarily explore the regulatory mechanism of PRDXs in HCC.

\section{Conclusions}

In summary, online tools were used to analyze and evaluate HCC data from public databases in this study, which provided multi-level evidence for the expression of PRDX family members in HCC and their predictive role in the prognosis of patients with HCC. The results showed that the deregulation of PRDXs in HCC played a vital role in genome stability, prognosis, cell pathway and function. The advantages of this study are large sample size, low cost and easy operation. Nonetheless, there are some limitations in this study that the data sources are relatively single: all the analyses in this study are based on the sequencing results of HCC patients in TCGA database. In addition, the results of analysis using different online tools are not entirely consistent, so our results should be further validated in experiments.

\section{Declarations}

\section{List of abbreviations}

Not applicable.

\section{Ethics approval and consent to participate}

Not applicable.

\section{Consent for publication}

Not applicable.

\section{Availability of data and materials}

Not applicable. 


\section{Competing interests}

The authors declare that they have no competing interests.

\section{Funding}

This study was supported by Science and Technology Planning Project of Guangdong Province, China (Grant No.2012A020602002).

\section{Author's contributions}

$R X, L L, S C$ and YL have made significant contribution to the concept and design of this study. MX, JX, DZ, $\mathrm{LL}$ and SC collected the data from several database, analyzed the data by using several online tools and collated analysis results. RS and BZ produced this manuscript. RX and YL reviewed the results and revised the manuscript. All authors read and approved the final manuscript.

\section{Acknowledgements}

This study was supported by Science and Technology Planning Project of Guangdong Province, China.

\section{References}

1. R. Xue, L. Chen, C. Zhang, M. Fujita, R. Li, S.M. Yan, et al. Genomic and Transcriptomic Profiling of Combined Hepatocellular and Intrahepatic Cholangiocarcinoma Reveals Distinct Molecular Subtypes. Cancer Cell. 2019, 35(6): 932-947.e8.

2. J.M. Llovet, R. Montal, D. Sia and R.S. Finn. Molecular therapies and precision medicine for hepatocellular carcinoma. Nat Rev Clin Oncol. 2018, 15(10): 599-616.

3. L. Kulik and H.B. El-Serag. Epidemiology and Management of Hepatocellular Carcinoma. Gastroenterology. 2019, 156(2): 477-491.e1.

4. T.F. Greten, C.W. Lai, G. Li and K.F. Staveley-O'Carroll. Targeted and Immune-Based Therapies for Hepatocellular Carcinoma. Gastroenterology. 2019, 156(2): 510-524.

5. A. Riaz, R. Lewandowski and R. Salem. Radioembolization in Advanced Hepatocellular Carcinoma. J Clin Oncol. 2018, 36(19): 1898-1901.

6. M. Lu, W.W. Zhu, X. Wang, J.J. Tang, K.L. Zhang, G.Y. Yu, et al. ACOT12-Dependent Alteration of Acetyl-CoA Drives Hepatocellular Carcinoma Metastasis by Epigenetic Induction of EpithelialMesenchymal Transition. Cell Metab. 2019, 29(4): 886-900.e5.

7. Y. Jiang, A. Sun, Y. Zhao, W. Ying, H. Sun, X. Yang, et al. Proteomics identifies new therapeutic targets of early-stage hepatocellular carcinoma. Nature. 2019, 567(7747): 257-261.

8. Z. Li, H. Zhang, J. Han, Y. Chen, H. Lin and T. Yang. Surface Nanopore Engineering of 2D MXenes for Targeted and Synergistic Multitherapies of Hepatocellular Carcinoma. Adv Mater. 2018, 30(25): e1706981. 
9. B. Knoops, V. Argyropoulou, S. Becker, L. Ferte and O. Kuznetsova. Multiple Roles of Peroxiredoxins in Inflammation. Mol Cells. 2016, 39(1): 60-4.

10. D. Yaron-Marcovich, I. Dror and B. Berkowitz. Behavior and stability of organic contaminant droplets in aqueous solutions. Chemosphere. 2007, 69(10): 1593-601.

11. B.L. Hopkins, M. Nadler, J.J. Skoko, T. Bertomeu, A. Pelosi, P.M. Shafaei, et al. A Peroxidase Peroxiredoxin 1-Specific Redox Regulation of the Novel FOX03 microRNA Target let-7. Antioxid Redox Signal. 2018, 28(1): 62-77.

12. S.J. Jeong, S. Kim, J.G. Park, I.H. Jung, M.N. Lee, S. Jeon, et al. Prdx1 (peroxiredoxin 1) deficiency reduces cholesterol efflux via impaired macrophage lipophagic flux. Autophagy. 2018, 14(1): 120133.

13. Y. Min, M.J. Kim, S. Lee, E. Chun and K.Y. Lee. Inhibition of TRAF6 ubiquitin-ligase activity by PRDX1 leads to inhibition of NFKB activation and autophagy activation. Autophagy. 2018, 14(8): 1347-1358.

14. M. Ying, X. Shao, H. Jing, Y. Liu, X. Qi, J. Cao, et al. Ubiquitin-dependent degradation of CDK2 drives the therapeutic differentiation of AML by targeting PRDX2. Blood. 2018, 131(24): 2698-2711.

15. Y. Yan, C. Wladyka, J. Fujii and S. Sockanathan. Prdx4 is a compartment-specific H2O2 sensor that regulates neurogenesis by controlling surface expression of GDE2. Nat Commun. 2015, 6:7006.

16. S.L. Zhou, Z.J. Zhou, Z.Q. Hu, C.L. Song, Y.J. Luo, C.B. Luo, et al. Genomic Sequencing Identifies WNK2 as a Driver in hepatocellular carcinoma and a Risk Factor for Early Recurrence. J Hepatol. 2019.

17. J. Cai, L. Chen, Z. Zhang, X. Zhang, X. Lu, W. Liu, et al. Genome-wide mapping of 5hydroxymethylcytosines in circulating cell-free DNA as a non-invasive approach for early detection of hepatocellular carcinoma. Gut. 2019.

18. J. Wang, J. Meng, W. Ran, R.J. Lee, L. Teng, P. Zhang, et al. Hepatocellular Carcinoma Growth Retardation and PD-1 Blockade Therapy Potentiation with Synthetic High-density Lipoprotein. Nano Lett. 2019, 19(8): 5266-5276.

19. M.B. Hampton, K.A. Vick, J.J. Skoko and C.A. Neumann. Peroxiredoxin Involvement in the Initiation and Progression of Human Cancer. Antioxid Redox Signal. 2018, 28(7): 591-608.

20. P. Aguilar-Melero, M.J. Prieto-Alamo, J. Jurado, A. Holmgren and C. Pueyo. Proteomics in HepG2 hepatocarcinoma cells with stably silenced expression of PRDX1. J Proteomics. 2013, 79: 161-71.

21. S. Zhou, Q. Han, R. Wang, X. Li, Q. Wang, H. Wang, et al. PRDX2 protects hepatocellular carcinoma SMMC-7721 cells from oxidative stress. Oncol Lett. 2016, 12(3): 2217-2221.

22. X. Xu, D. Lu, R. Zhuang, X. Wei, H. Xie, C. Wang, et al. The phospholipase A2 activity of peroxiredoxin 6 promotes cancer cell death induced by tumor necrosis factor alpha in hepatocellular carcinoma. Mol Carcinog. 2016, 55(9): 1299-308.

23. Y. Fang, J. He, H.L.A. Janssen, J. Wu, L. Dong and X.Z. Shen. Peroxiredoxin 1, restraining cell migration and invasion, is involved in hepatocellular carcinoma recurrence. J Dig Dis. 2018, 19(3): 155-169. 
24. G.Q. Zhu, Y. Yang, E.B. Chen, B. Wang, K. Xiao, S.M. Shi, et al. Development and validation of a new tumor-based gene signature predicting prognosis of HBV/HCV-included resected hepatocellular carcinoma patients. J Transl Med. 2019, 17(1): 203.

25. D. Ji, M. Li, T. Zhan, Y. Yao, J. Shen, H. Tian, et al. Prognostic role of serum AZGP1, PEDF and PRDX2 in colorectal cancer patients. Carcinogenesis. 2013, 34(6): 1265-72.

26. X. Guo, H. Noguchi, N. Ishii, T. Homma, T. Hamada, T. Hiraki, et al. The Association of Peroxiredoxin 4 with the Initiation and Progression of Hepatocellular Carcinoma. Antioxid Redox Signal. 2019, 30(10): 1271-1284.

27. I. Annunziata, D. van de Vlekkert, E. Wolf, D. Finkelstein, G. Neale, E. Machado, et al. MYC competes with MiT/TFE in regulating lysosomal biogenesis and autophagy through an epigenetic rheostat. Nat Commun. 2019, 10(1): 3623.

28. L. Chang, D. Zhang, H. Shi, Y. Bian and R. Guo. MiR-143 inhibits endometrial cancer cell proliferation and metastasis by targeting MAPK1. Oncotarget. 2017, 8(48): 84384-84395.

29. Y.F. Yang, Y.H. Pan, Y. Cao, J. Fu, X. Yang, M.F. Zhang, et al. PDZ binding kinase, regulated by FoxM1, enhances malignant phenotype via activation of beta-Catenin signaling in hepatocellular carcinoma. Oncotarget. 2017, 8(29): 47195-47205.

30. H. Ma, Y. Li, X. Wang, H. Wu, G. Qi, R. Li, et al. PBK, targeted by EVI1, promotes metastasis and confers cisplatin resistance through inducing autophagy in high-grade serous ovarian carcinoma. Cell Death Dis. 2019, 10(3): 166.

31. M.J. Zheng, J. Wang, H.M. Wang, L.L. Gao, X. Li, W.C. Zhang, et al. Decreased expression of peroxiredoxin 1 inhibits proliferation, invasion, and metastasis of ovarian cancer cell. Onco Targets Ther. 2018, 11: 7745-7761.

32. R. Wang, J. Wei, S. Zhang, X. Wu, J. Guo, M. Liu, et al. Peroxiredoxin 2 is essential for maintaining cancer stem cell-like phenotype through activation of Hedgehog signaling pathway in colon cancer. Oncotarget. 2016, 7(52): 86816-86828.

33. Z. Lv, J. Wei, W. You, R. Wang, J. Shang, Y. Xiong, et al. Disruption of the c-Myc/miR-200b-3p/PRDX2 regulatory loop enhances tumor metastasis and chemotherapeutic resistance in colorectal cancer. J Transl Med. 2017, 15(1): 257.

34. K.K. Li, J.C. Pang, K.M. Lau, L. Zhou, Y. Mao, Y. Wang, et al. MiR-383 is downregulated in medulloblastoma and targets peroxiredoxin 3 (PRDX3). Brain Pathol. 2013, 23(4): 413-25.

\section{Tables}

Table 1. The type and frequency of PRDXs gene family neighbor gene alterations in hepatocellular carcinoma (cBioPortal). 


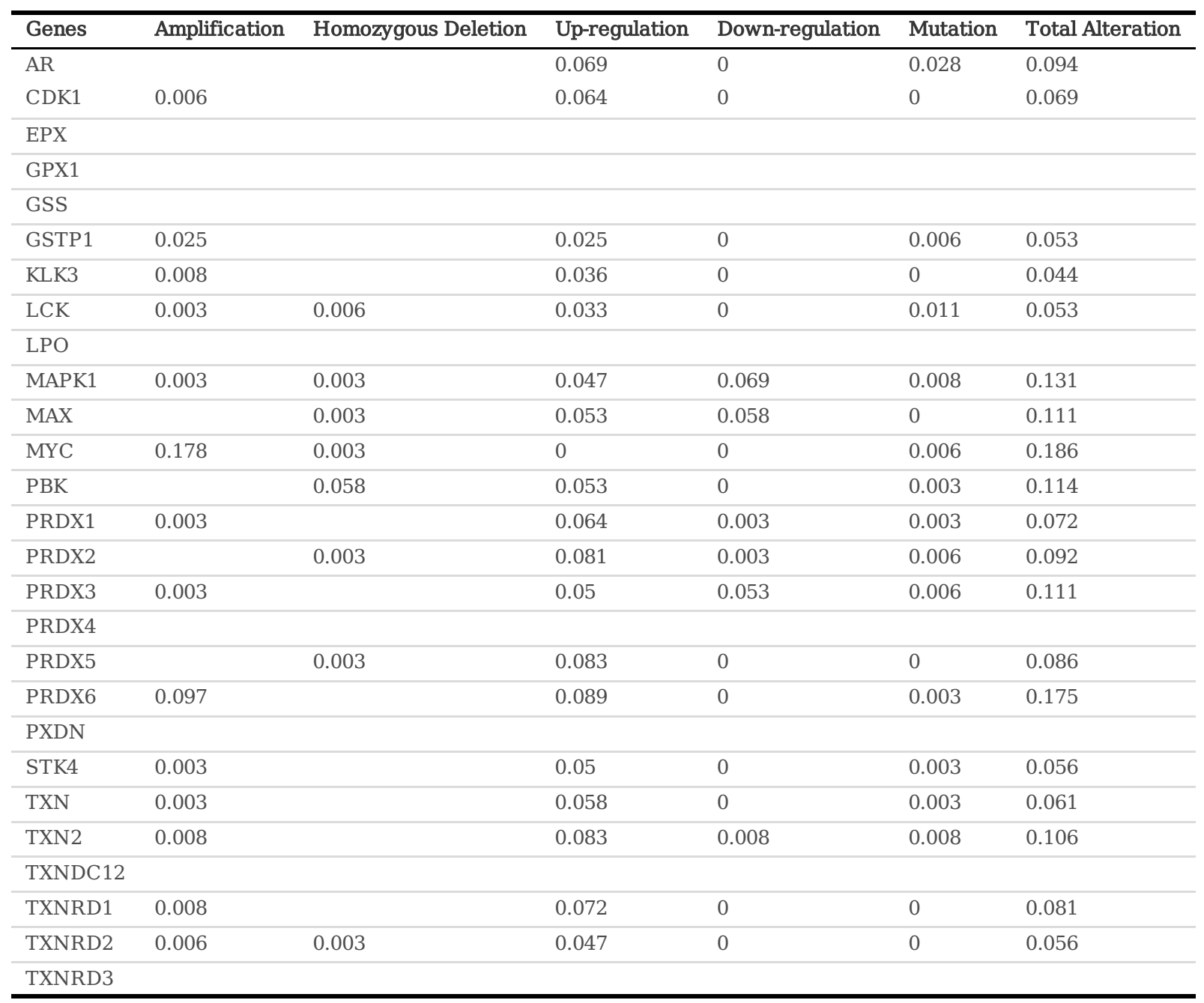

\section{Supplemental Information Note}

\section{Supplementary figure 1}

The mRNA expression levels of PRDX3 (A) and PRDX4 (B) in a variety of cancer cell lines were obtained from CCLE database.

\section{Supplementary figure 2}

The mRNA expression levels of PRDX5 (A) and PRDX6 (B) in a variety of cancer cell lines were obtained from CCLE database.

\section{Supplementary figure 3}

The mRNA expression levels of PRDX2 (A) and PRDX3 (B) gene family in different HCC datasets of HCCDB database were analyzed. Red: HCC samples; blue: adjacent normal tissue samples; cyan: cirrhotic samples; orange: healthy samples. 


\section{Supplementary figure 4}

The mRNA expression levels of PRDX4 (A) and PRDX6 (B) gene family in different HCC datasets of HCCDB database were analyzed. Red: HCC samples; blue: adjacent normal tissue samples; cyan: cirrhotic samples; orange: healthy samples.

Supplementary figure 5 KEGG pathway enrichment analysis of PRDX2 co-expression genes and miRNA targets of PRDX2 in HCC

(A) Volcano plot showed the differential expression of genes related to PRDX2 in HCC and a Pearson correlation was used for the correlation analysis. Green: negatively correlated significant genes; red: positively correlated significant genes. (B) Top 50 positively correlated significant genes of PRDX2 were presented in the heat map. (C) Top 50 negatively correlated significant genes of PRDX2 were presented in the heat map. (D) The KEGG pathway enrichment of PRDX2 co-expression genes in HCC was analyzed using GSEA. (E) The miRNA targets of PRDX2 in HCC. FDR: false discovery rate.

Supplementary figure 6 KEGG pathway enrichment analysis of PRDX3 co-expression genes and miRNA targets of PRDX3 in HCC

(A) Volcano plot showed the differential expression of genes related to PRDX3 in HCC and a Pearson correlation was used for the correlation analysis. Green: negatively correlated significant genes; red: positively correlated significant genes. (B) Top 50 positively correlated significant genes of PRDX3 were presented in the heat map. (C) Top 50 negatively correlated significant genes of PRDX3 were presented in the heat map. (D) The KEGG pathway enrichment of PRDX3 co-expression genes in HCC was analyzed using GSEA. (E) The miRNA targets of PRDX3 in HCC. FDR: false discovery rate.

Supplementary figure 7 KEGG pathway enrichment analysis of PRDX4 co-expression genes and miRNA targets of PRDX4 in HCC

(A) Volcano plot showed the differential expression of genes related to PRDX4 in HCC and a Pearson correlation was used for the correlation analysis. Green: negatively correlated significant genes; red: positively correlated significant genes. (B) Top 50 positively correlated significant genes of PRDX4 were presented in the heat map. (C) Top 50 negatively correlated significant genes of PRDX4 were presented in the heat map. (D) The KEGG pathway enrichment of PRDX4 co-expression genes in HCC was analyzed using GSEA. (E) The miRNA targets of PRDX4 in HCC. FDR: false discovery rate.

Supplementary figure 8 KEGG pathway enrichment analysis of PRDX5 co-expression genes and miRNA targets of PRDX5 in HCC

(A) Volcano plot showed the differential expression of genes related to PRDX5 in HCC and a Pearson correlation was used for the correlation analysis. Green: negatively correlated significant genes; red: positively correlated significant genes. (B) Top 50 positively correlated significant genes of PRDX5 were presented in the heat map. (C) Top 50 negatively correlated significant genes of PRDX5 were presented in 
the heat map. (D) The KEGG pathway enrichment of PRDX5 co-expression genes in HCC was analyzed using GSEA. (E) The miRNA targets of PRDX5 in HCC. FDR: false discovery rate.

Supplementary figure 9 KEGG pathway enrichment analysis of PRDX6 co-expression genes and miRNA targets of PRDX6 in HCC

(A) Volcano plot showed the differential expression of genes related to PRDX6 in HCC and a Pearson correlation was used for the correlation analysis. Green: negatively correlated significant genes; red: positively correlated significant genes. (B) Top 50 positively correlated significant genes of PRDX6 were presented in the heat map. (C) Top 50 negatively correlated significant genes of PRDX6 were presented in the heat map. (D) The KEGG pathway enrichment of PRDX6 co-expression genes in HCC was analyzed using GSEA. (E) The miRNA targets of PRDX6 in HCC. FDR: false discovery rate.

\section{Figures}


A

PRDX1 - Entrez ID: 5052

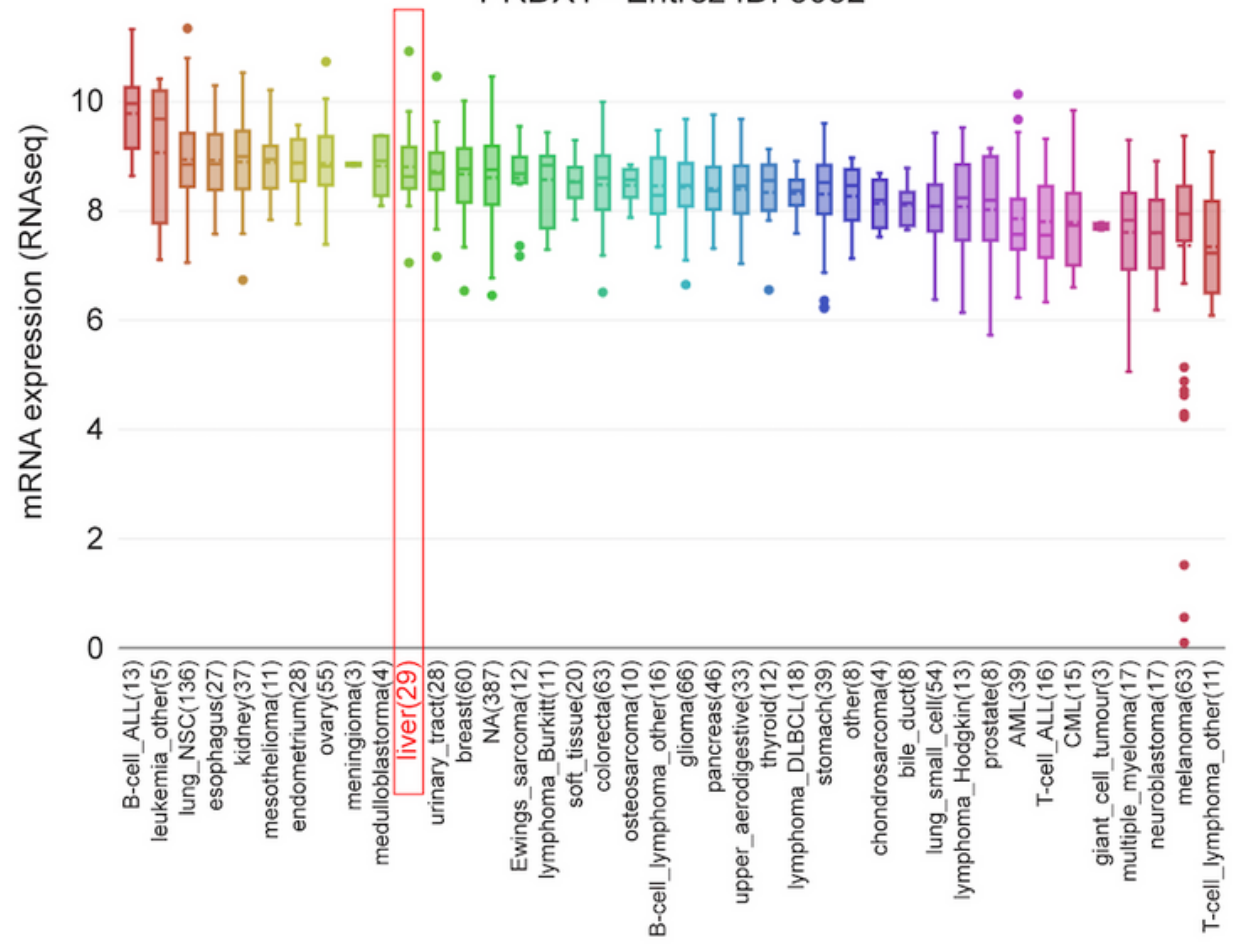

B $10 \quad$ PRDX2 - Entrez ID: 7001

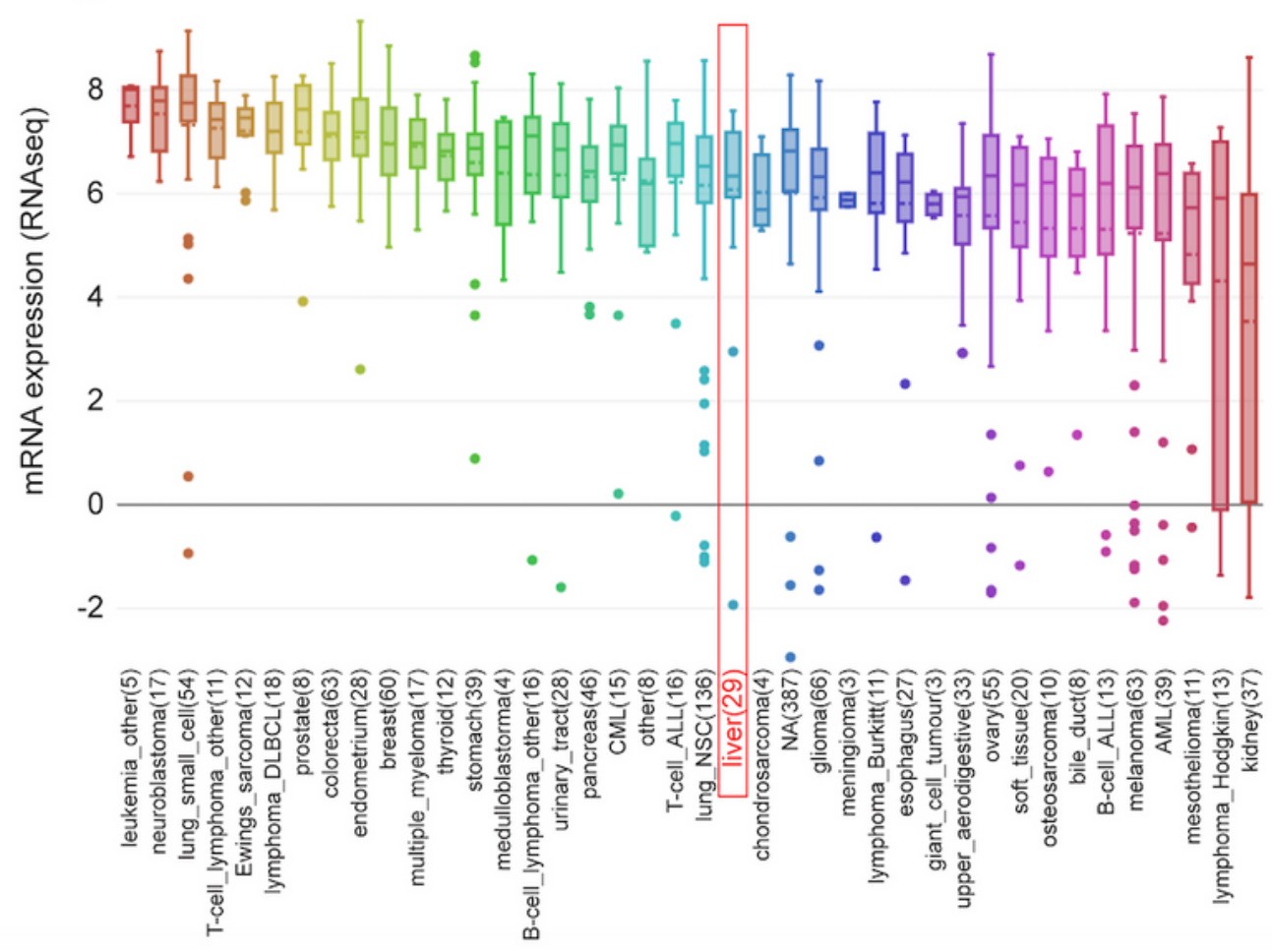

Figure 1

The mRNA expression levels of PRDX1 (A) and PRDX2 (B) in multiple common cancer cell lines were obtained from CCLE database. The expression of these two genes in hepatocellular carcinoma (HCC) was shown in red frame. 
Figure 2

The protein levels of PRDXs in HCC tissues by Human Protein Atlas database The protein levels of PRDXs (PRDX1-6) in HCC tissues were compared with that in normal tissues by immunohistochemical staining, shown in panel (A-F).

A

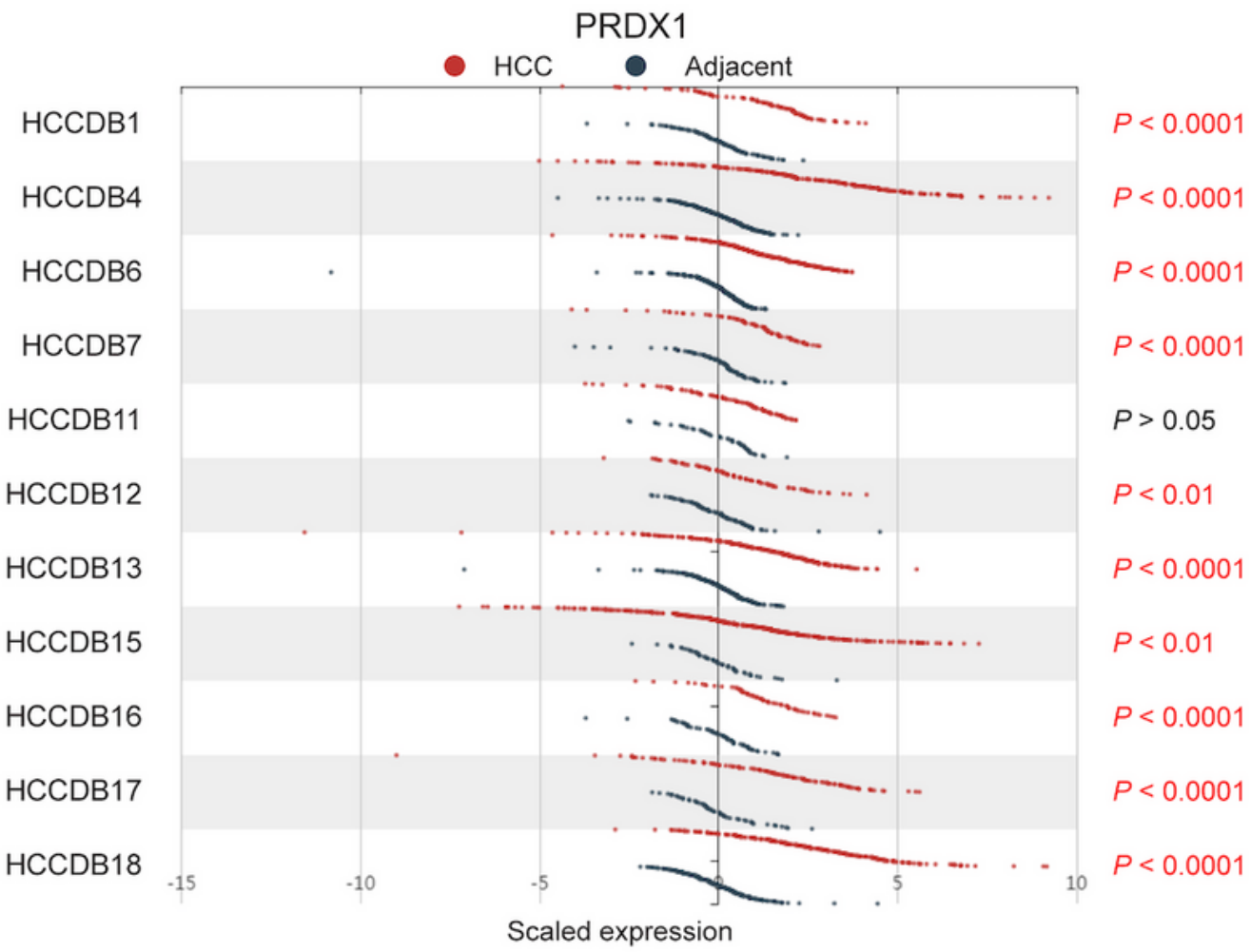

B

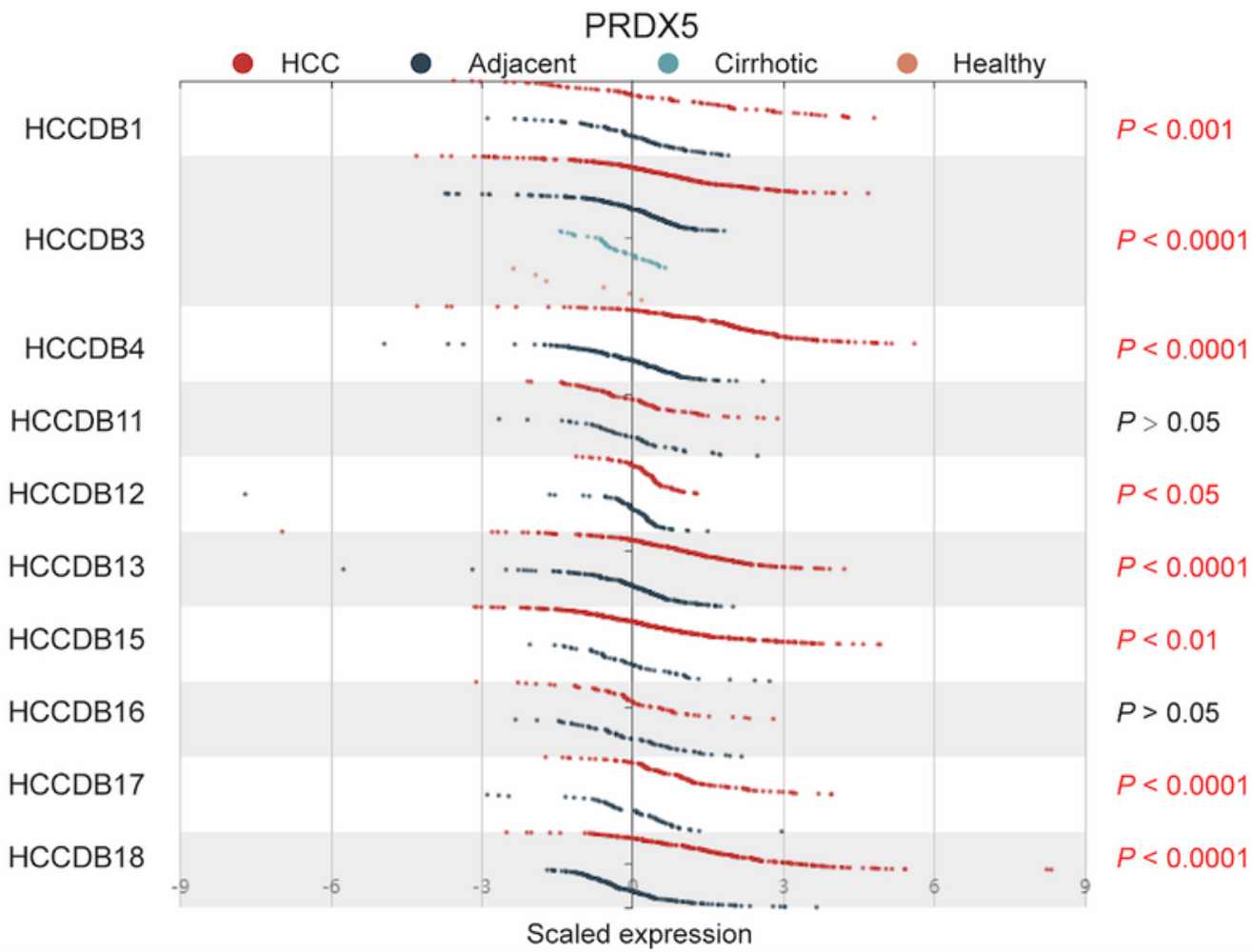

Figure 3 
The mRNA expression levels of PRDX1 (A) and PRDX5 (B) in different HCC datasets of HCCDB database were analyzed. Red: HCC samples; blue: adjacent normal tissue samples; cyan: cirrhotic samples; orange: healthy samples.

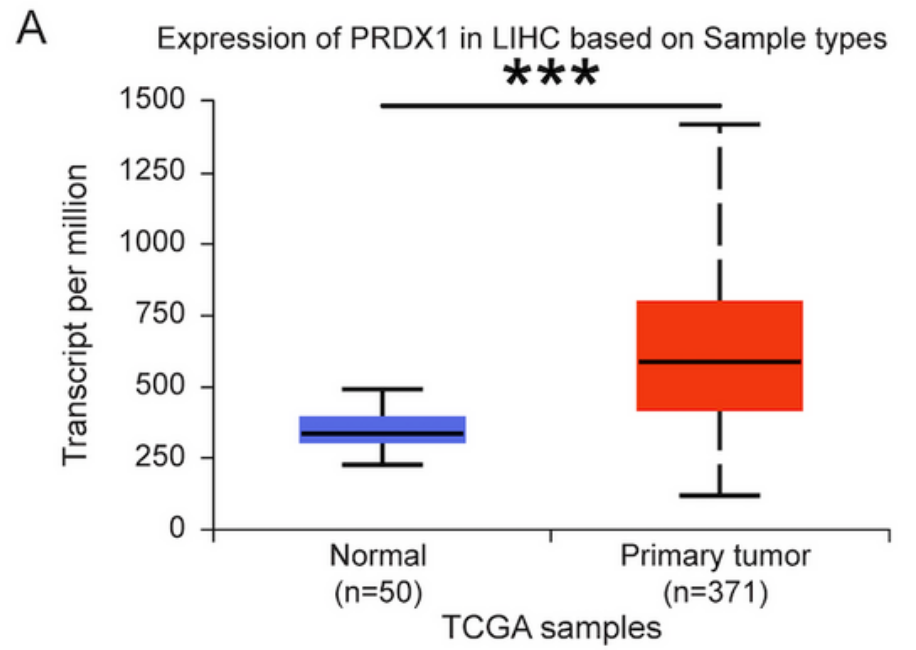

C Expression of PRDX3 in LIHC based on Sample types

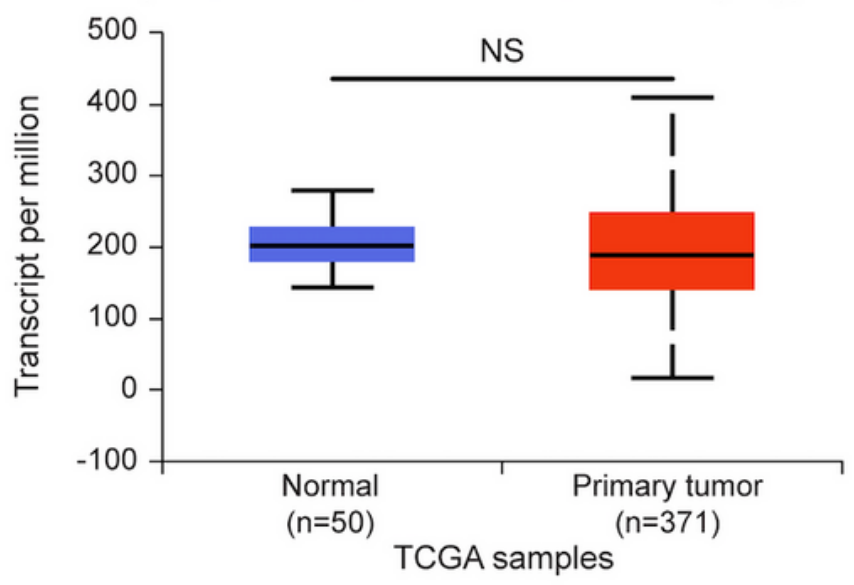

$E$

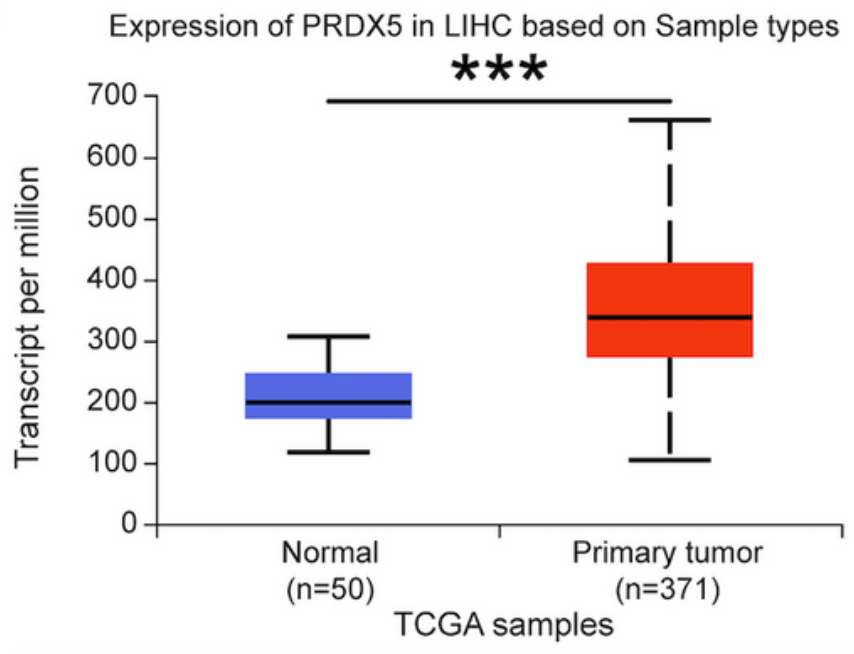

B Expression of PRDX2 in LIHC based on Sample types

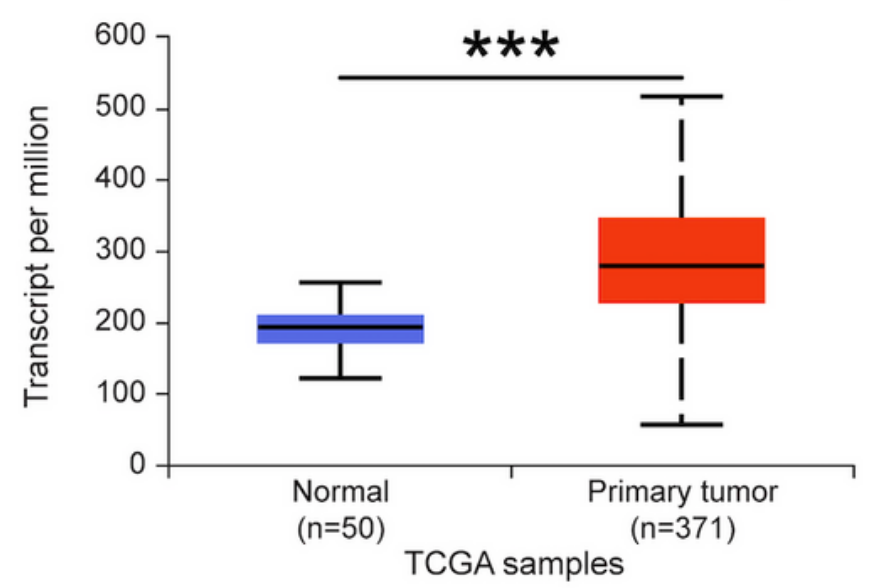

D

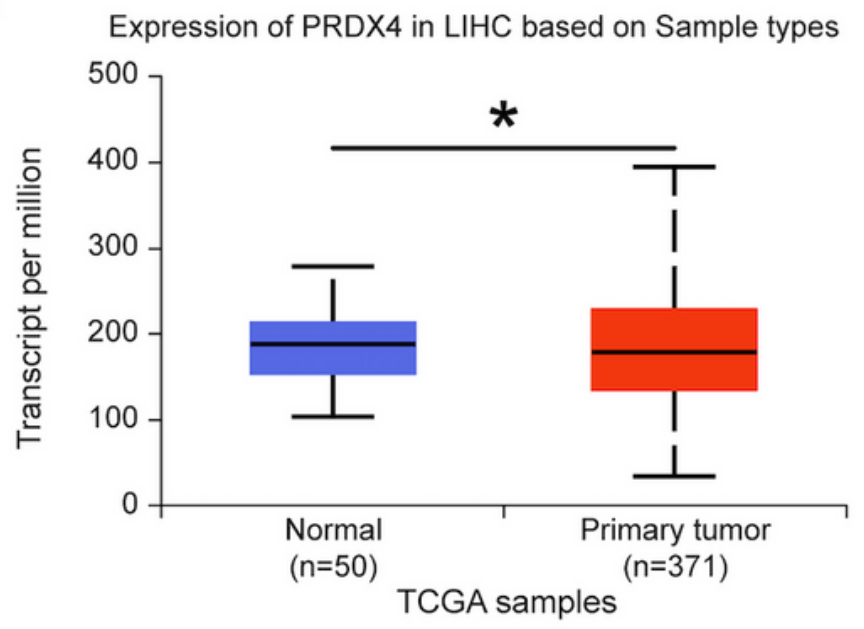

$\mathrm{F}$

Expression of PRDX6 in LIHC based on Sample types

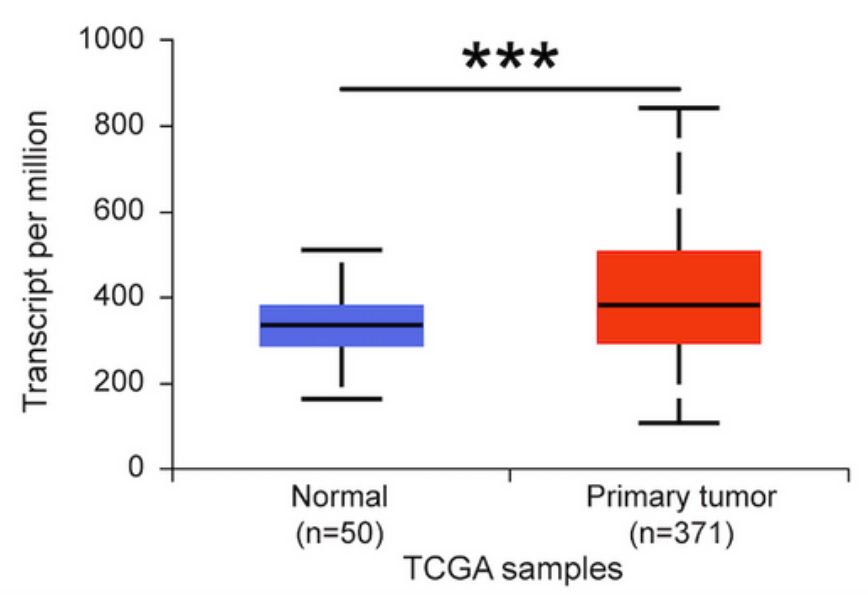

Figure 4

Boxplot showed the relative expression of PRDXs in normal and HCC samples (UALCAN) Panel (A-F) represented for PRDX1, PRDX2, PRDX3, PRDX4, PRDX5, and PRDX6 mRNA expression in HCC samples 
relative to normal samples based on TCGA database. LIHC: the abbreviation of liver hepatocellular carcinoma in TCGA database. * $\mathrm{P}<0.05$; ** $\mathrm{P}<0.0 .1$; *** $\mathrm{P}<0.001$.

A

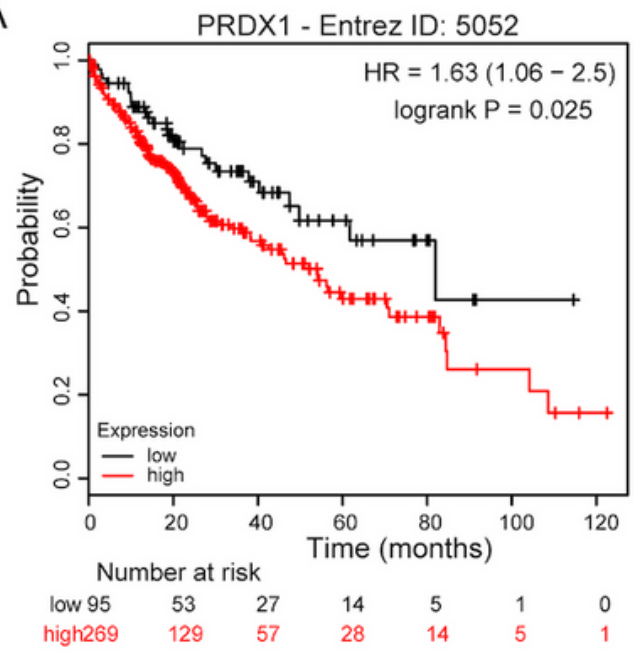

C

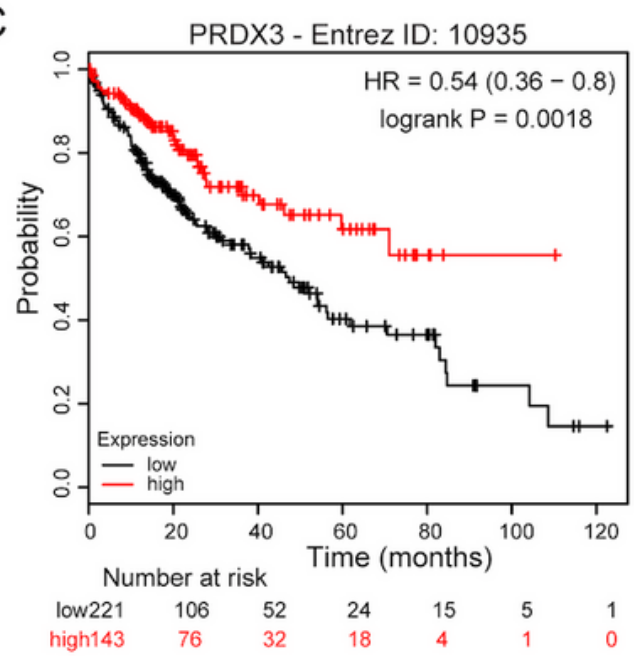

E

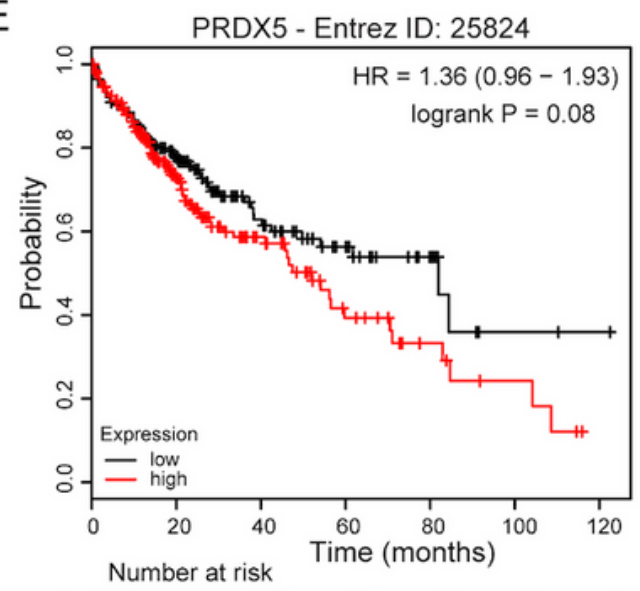

$\mathrm{B}$

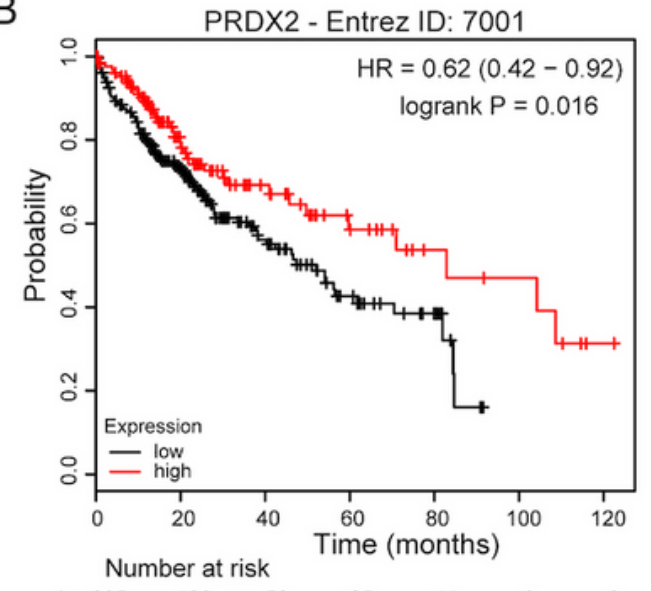

D PRDX4 - Entrez ID: 10549

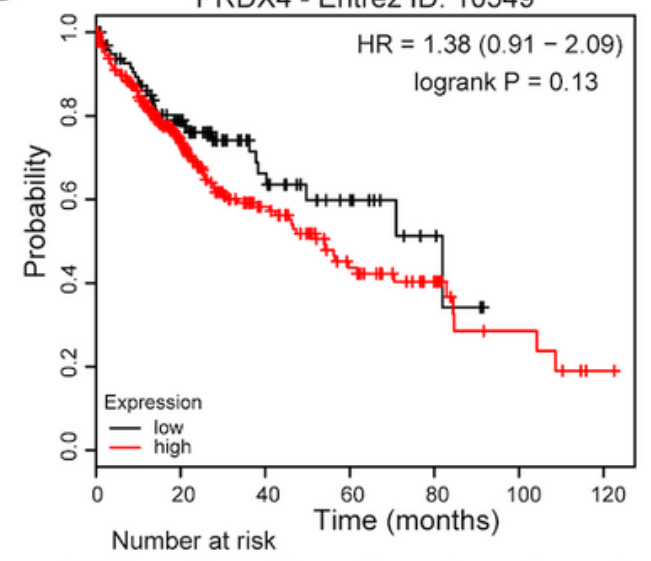

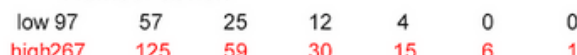

$\mathrm{F}$

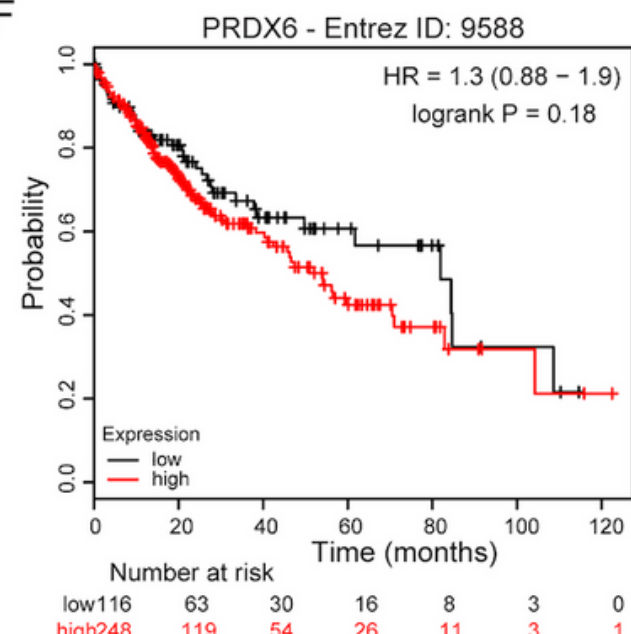

Figure 5

The correlations between PRDXs expression and patient prognosis in HCC Kaplan-Meier Plotter database was utilized to evaluate the correlations between the expression of PRDXs and prognosis of HCC patients $(\mathrm{A}-\mathrm{F})$. 


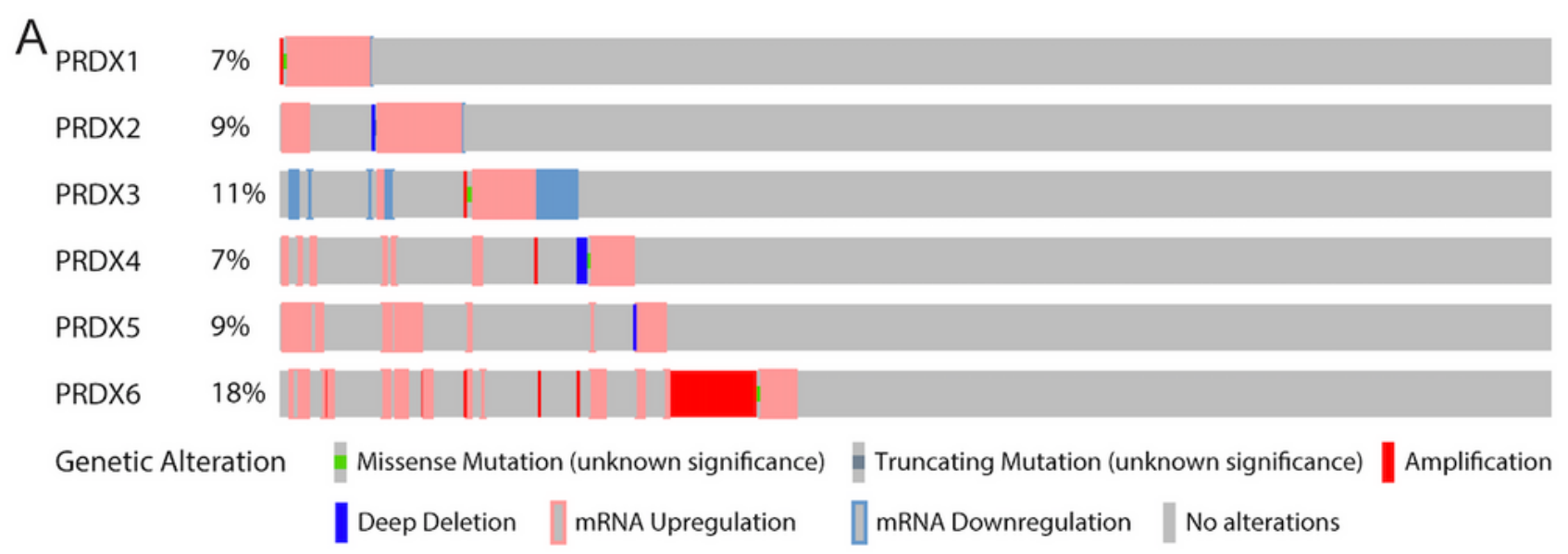

B

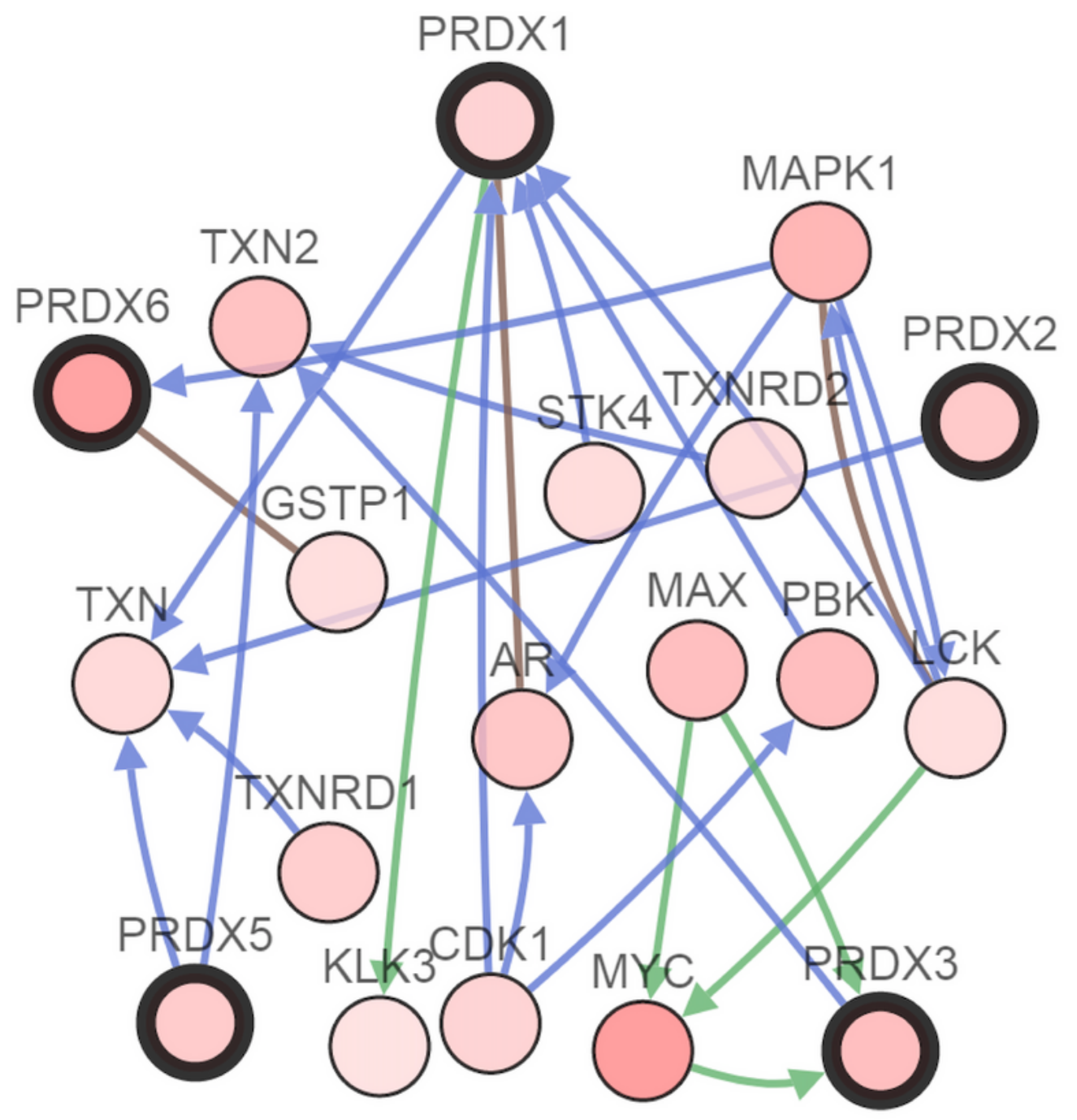

\section{Figure 6}

PRDXs alterations and biological interaction network in HCC (A) The genetic alteration of PRDXs in HCC patients from TCGA were analyzed by c-BioPortal database. The different types of genetic alteration were highlighted in different color. (B) The network of PRDXs neighbor genes in HCC. PRDX1, PRDX2, PRDX3, PRDX5, and PRDX6 were the seed genes, indicated with thick border. The lighter red color suggested decrease frequency of genetic alteration in HCC. 


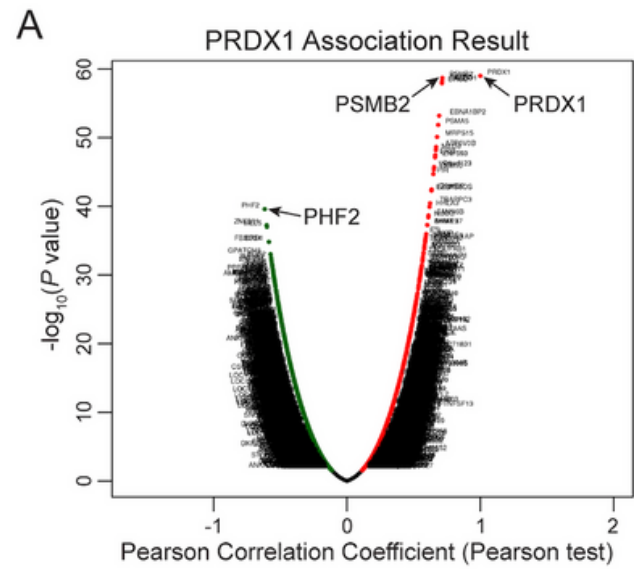

D

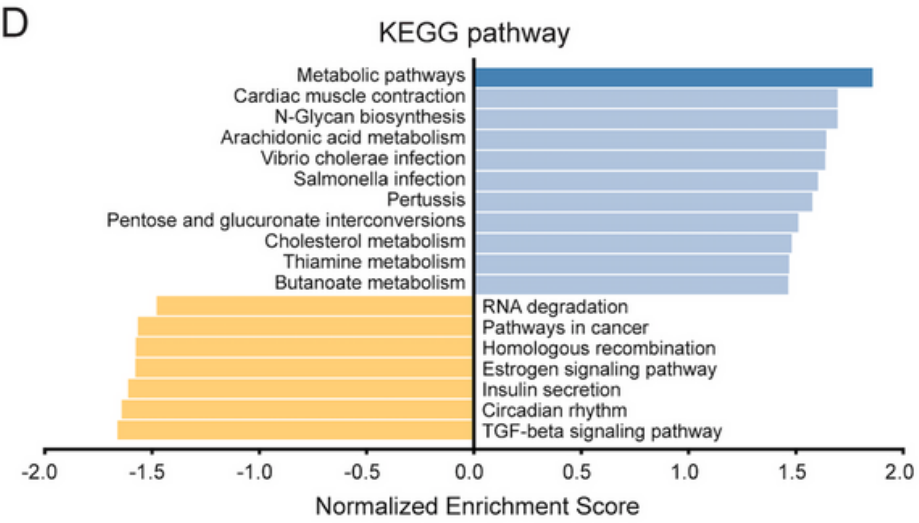

B

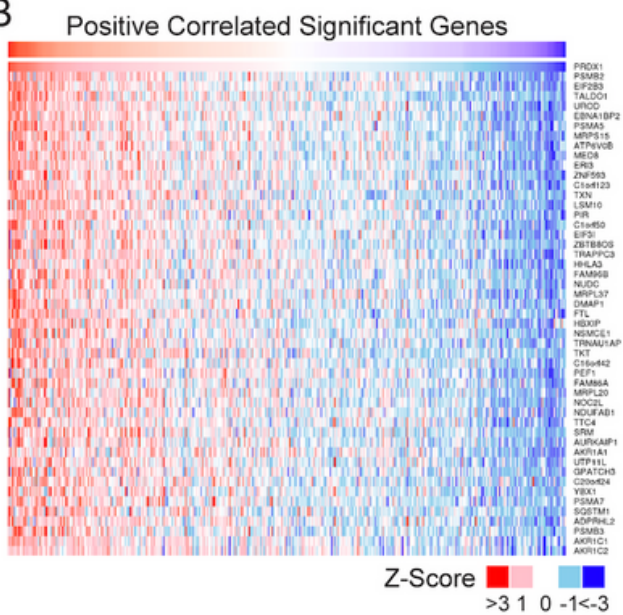

$\mathrm{E}$
C

Negetive Correlated Significant Genes

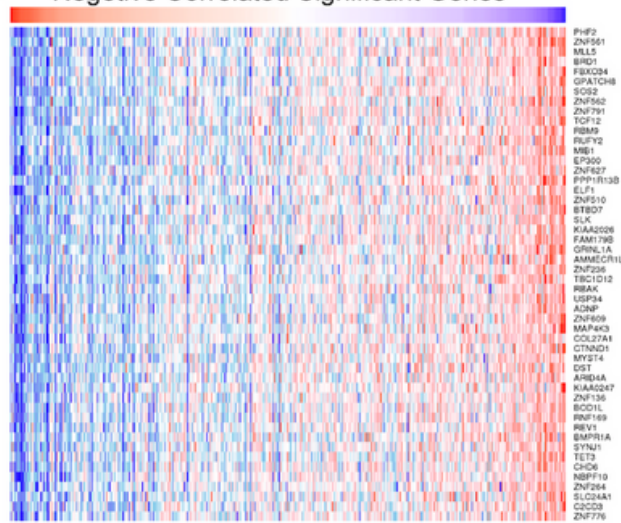

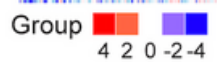

miRNA targets of PRDX1

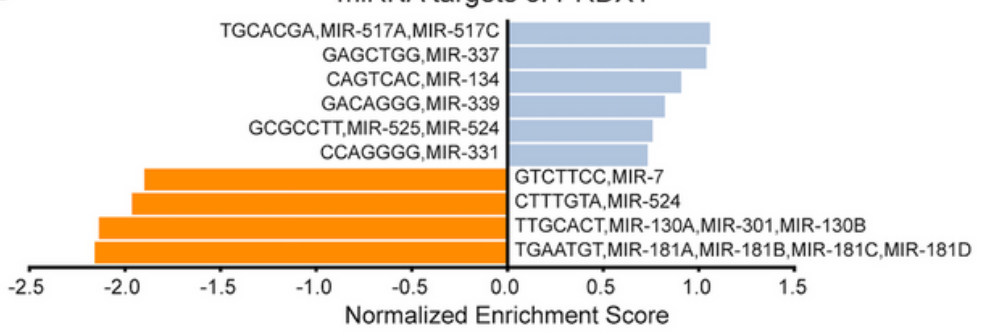

FDR $\leq 0.05=$ FDR $>0.05$

\section{Figure 7}

KEGG pathway enrichment analysis of PRDX1 co-expression genes and miRNA targets of PRDX1 in HCC (A) Volcano plot showed the differential expression of genes related to PRDX1 in HCC and a Pearson correlation was used for the correlation analysis. Green: negatively correlated significant genes; red: positively correlated significant genes. (B) Top 50 positively correlated significant genes of PRDX1 were presented in the heat map. (C) Top 50 negatively correlated significant genes of PRDX1 were presented in the heat map. (D) The KEGG pathway enrichment of PRDX1 co-expression genes in HCC was analyzed using Gene Set Enrichment Analysis (GSEA). (E) The miRNA targets of PRDX1 in HCC. FDR: false discovery rate.

\section{Supplementary Files}

This is a list of supplementary files associated with this preprint. Click to download.

- Supplementaryfigure1.tif

- Supplementarytable7.docx

- Supplementaryfigure9.tif

- Supplementarytable1.docx

- Supplementarytable2.docx 
- Supplementarytable3.docx

- Supplementarytable4.docx

- Supplementarytable5.docx

- Supplementarytable6.docx

- Supplementaryfigure8.tif

- Supplementaryfigure6.tif

- Supplementaryfigure7.tif

- Supplementaryfigure5.tif

- Supplementaryfigure3.tif

- Supplementaryfigure4.tif

- Supplementaryfigure2.tif 